ARTICLE

https://doi.org/10.1038/s41467-019-09141-w

\title{
Sperm-inherited H3K27me3 impacts offspring transcription and development in C. elegans
}

\author{
Kiyomi Raye Kaneshiro ${ }^{1}$, Andreas Rechtsteiner ${ }^{1} \&$ Susan Strome (iD ${ }^{1}$
}

Paternal epigenetic inheritance is gaining attention for its growing medical relevance. However, the form in which paternal epigenetic information is transmitted to offspring and how it influences offspring development remain poorly understood. Here we show that in C. elegans, sperm-inherited chromatin states transmitted to the primordial germ cells in offspring influence germline transcription and development. We show that sperm chromosomes inherited lacking the repressive histone modification $\mathrm{H} 3 \mathrm{~K} 27 \mathrm{me} 3$ are maintained in that state by H3K36me3 antagonism. Inheritance of H3K27me3-lacking sperm chromosomes results in derepression in the germline of somatic genes, especially neuronal genes, predominantly from sperm-inherited alleles. This results in germ cells primed for losing their germ cell identity and adopting a neuronal fate. These data demonstrate that histone modifications are one mechanism through which epigenetic information from a father can shape offspring gene expression and development.

\footnotetext{
${ }^{1}$ Department of Molecular, Cell and Developmental Biology, University of California, Santa Cruz, 1156 High Street, Santa Cruz, CA 95064, USA. Correspondence and requests for materials should be addressed to S.S. (email: sstrome@ucsc.edu)
} 
T here is growing awareness that development and health are influenced by epigenetic information passed from parents to offspring ${ }^{1,2}$. The gametes, sperm and oocyte, are the conduits through which epigenetic information is passed from one generation to the next. Investigating epigenetic inheritance via the maternal line is complicated by the many non-epigenetic factors the oocyte provides to the embryo ${ }^{1}$. For this reason, the field of epigenetic inheritance largely focuses on transmission via sperm. DNA methylation and non-coding RNAs (ncRNAs) are epigenetic carriers that are transmitted by sperm to the embryo and that have been shown to influence gene expression in offspring ${ }^{3,4}$. Modified histones can also be passed, but their role in intergenerational and transgenerational epigenetic inheritance is less clear. In mammals, the sperm genome is primarily packaged with protamines and, while there is current debate about the degree to which the sperm genome retains histones, there is consensus that marked histones are found on developmentally important loci ${ }^{5-7}$. Despite this observation and mounting suggestive evidence ${ }^{8-10}$, establishing a causal role for sperminherited histone marks in regulating offspring transcription and development has been an ongoing challenge. We took advantage of features of $C$. elegans to tackle this issue.

We previously showed that $C$. elegans sperm retain nucleosomes and histone marking genome-wide ${ }^{11}$ and that C. elegans Polycomb Repressive Complex 2 (PRC2) maintains inherited states of $\mathrm{H} 3 \mathrm{~K} 27 \mathrm{me} 3$ during embryogenesis ${ }^{12}$. In wild-type embryos H3K27me3 is enriched over genes that were silent in the parental germline ${ }^{13,14}$. H2K27me3 marking inherited from hermaphrodite parent worms is essential for germline development in offspring, since hermaphrodite parents lacking PRC2 generate offspring in which the primordial germ cells die during early larval development ${ }^{15}$. We thus hypothesize that transmission of chromatin states from parent germ cells via sperm and oocyte to the nascent germ cells in offspring protects germlineappropriate gene expression patterns in the developing and adult germline.

In this work we investigate how chromatin states inherited from parents are maintained in offspring and whether inherited states are important for offspring transcription and development. We elucidate a mechanism through which an inherited H3K27me3(-) state is propagated from parent germ cells (sperm) to offspring germ cells. We show that inheriting a sperm genome lacking the repressive mark H3K27me3 results in derepression of many genes for somatic development, especially neuronal genes, in offspring germlines. This results in germ cells that in a sensitized genetic background lose their germ cell identity and adopt a neuronal fate. Taken together, these findings establish a cause-effect relationship between sperm-inherited histone marks and offspring transcription and development in $C$. elegans.

\section{Results}

Maintenance of an inherited $\mathrm{H} 3 \mathrm{~K} 27 \mathrm{me} 3(-)$ state requires MES-4. When $C$. elegans embryos inherit some chromosomes with and some chromosomes without H3K27me3, PRC2 maintains inherited states by (1) restoring levels of H3K27me3 on H3K27me3(+) chromosomes after DNA replication and (2) failing to de novo methylate H3K27me3(-) chromosomes $^{12}$. The ability of PRC2 to restore levels of H3K27me3 after genome duplication is likely explained by the EED subunit of PRC2 (MES-6 in worms) binding to H3K27me3 and stimulating the methyltransferase activity of the EZH2 subunit (MES-2 in worms $)^{16,17}$. How PRC2 is prevented from de novo methylating chromosomes inherited lacking H3K27me3 is less clear. One possibility is that chromosomes lacking $\mathrm{H} 3 \mathrm{~K} 27$ me3 are unable to recruit and stimulate PRC2 activity. Another possibility is that these chromosomes bear an opposing mark that antagonizes PRC2 activity. Likely candidates for antagonizing PRC2 activity are histone marks associated with gene expression, hereafter referred to as active marks, and their corresponding histone modifiers ${ }^{18,19}$.

To test if active marks prevent PRC2 from de novo methylating sperm-inherited H3K27me3(-) chromosomes during early embryogenesis, we monitored sperm chromosomes through rounds of cell division in embryos lacking a maternal load of MES-4 or SET-2, which generate the active marks H3K36me2/ $3^{13,20}$ and H3K4me2/321, respectively. We generated embryos that inherited $\mathrm{H} 3 \mathrm{~K} 27 \mathrm{me} 3(+)$ oocyte chromosomes and H3K27me3(-) sperm chromosomes by mating wild-type females with PRC2 mutant males (Fig. 1a). To increase the likelihood that sperm chromosomes completely lacked $\mathrm{H} 3 \mathrm{~K} 27 \mathrm{me} 3$, we used PRC2 mutant males whose parents also lacked PRC2. We call the offspring of wild-type mothers and PRC2 mutant fathers K27me3 $M+P-(\mathrm{M}+$ for maternal H3K27me3(+), P- for paternal H3K27me3(-)). We assessed whether sperm-inherited chromosomes retained the H3K27me3(-) state or acquired H3K27me3, by tracking the status of $\mathrm{H} 3 \mathrm{~K} 27 \mathrm{me} 3$ on sperm-inherited chromosomes during early embryogenesis. To facilitate analysis of sperm vs. oocyte chromosomes, we performed this analysis in a temperature-sensitive plk-1 mutant background that keeps sperm-inherited and oocyte-inherited chromosomes in separate nuclei for many divisions ${ }^{22}$ (Fig. 1b).

In all 2-cell K27me3 $M+P-$ plk-1 embryos, we observed that one nucleus in each cell contained all H3K27me3(+) chromosomes (oocyte-inherited) and the other nucleus contained H3K27me3(-) chromosomes (sperm-inherited). In control and SET-2-lacking embryos, this pattern persisted in all cells during the early divisions and in the P lineage (germ lineage) throughout embryogenesis (Fig. 1c, d, Supplementary Fig. 1). In contrast, in embryos depleted of MES-4, we observed gradual acquisition of H3K27me3 on the entire set of sperm chromosomes (Fig. 1c, Supplementary Fig. 1). To assess the appearance of H3K27me3 on sperm chromosomes, we analyzed K27me3 $M+P$ - embryos from mothers that were either mutant or wild type for mes-4 (see methods). At the 1-cell stage, all K27me3 $M+P$ - embryos examined contained sperm chromosomes lacking detectable H3K27me3 irrespective of maternal MES-4 $(n=6$ with maternal MES- 4 and $n=4$ without maternal MES- 4 ). In the presence of maternal MES-4, this state was maintained in all embryos up to the 4 -cell stage $(n=21)$ and in most embryos up to the 40 -cell stage $(n=15 / 19)$. In the absence of maternal MES-4, all 2-cell embryos examined acquired some H3K27me3 on sperm chromosomes $(n=6)$. The levels of H3K27me3 on sperm and oocyte chromosomes became indistinguishable in embryos by the 4-cell stage $(n=4)$ and at later stages $(n=15)$. This gradual acquisition of H3K27me3 matches the gradual loss of H3K $36 \mathrm{me} 3$ in the absence of maternal MES- $4^{23}$. Thus, MES-4 inhibits PRC2 activity, presumably through MES-4-mediated methylation of H3K36, to maintain the paternal H3K27me3(-) state. These findings show that the H3K27me3(-) state is maintained by opposing PRC2 activity with active marks and also suggest that PRC2 does not require H3K27me3 to seed its activity, as observed in mouse embryonic stem cells ${ }^{24}$.

Sperm chromatin states shape transcription in offspring. Do gamete-inherited chromatin states influence gene expression in offspring? This is a burning question in the field and one that remains elusive, in part because it is difficult to determine cause versus consequence when comparing chromatin states with transcriptional status. To establish a cause-effect relationship 
a

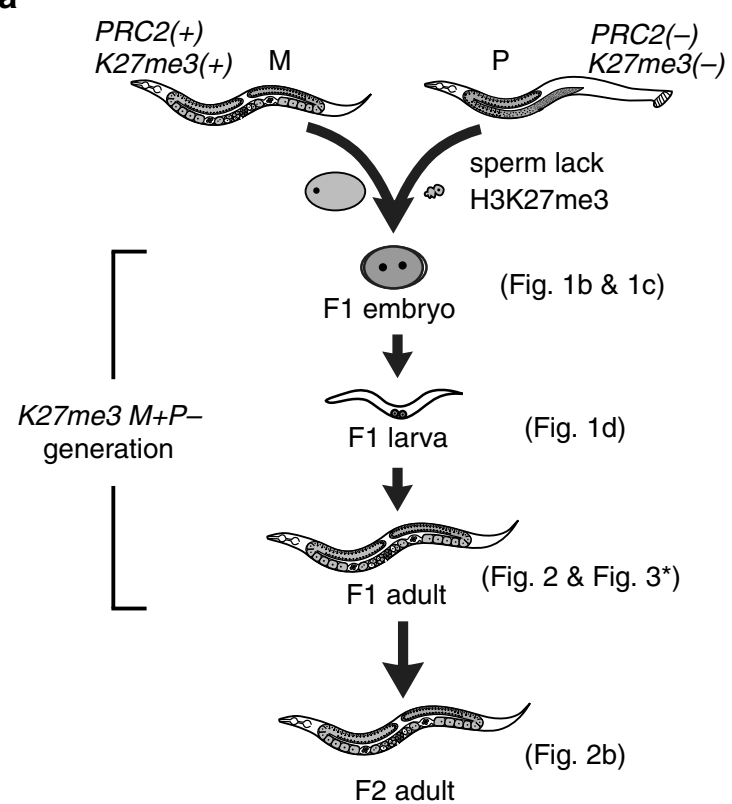

b

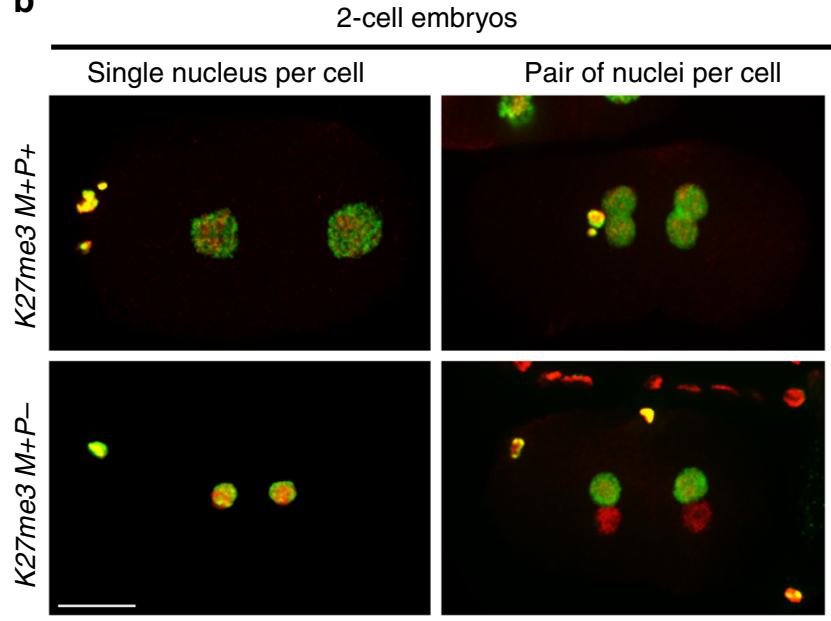

C Empty vector RNAi K27me3 $M+P$-embryos

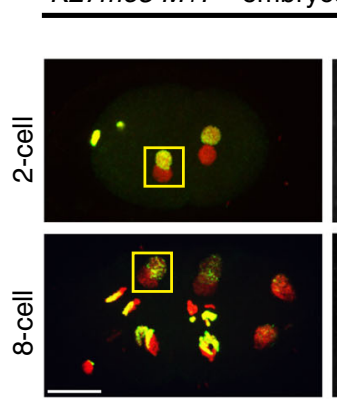

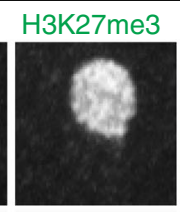
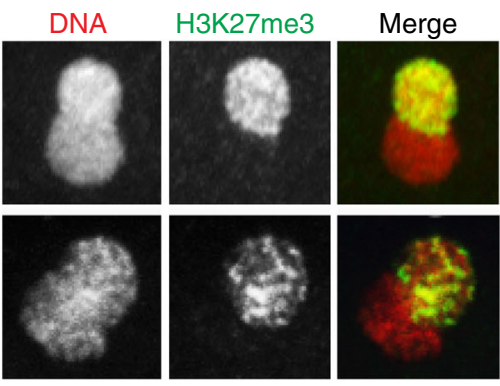

d DNA H3K27me3

\section{Merge}

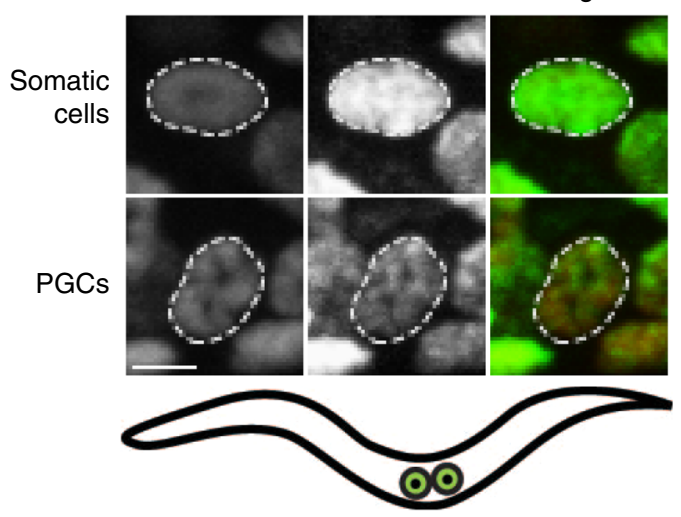

K27me3 $M+P+$ larva

mes-4 (RNAi)

K27me3 $M+P$-embryos

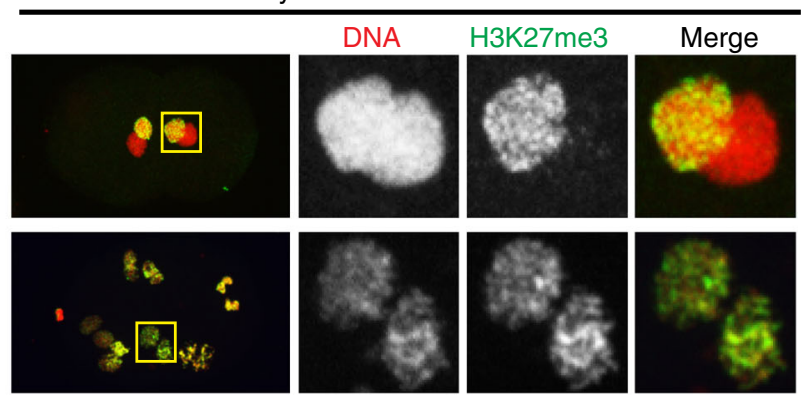

Fig. 1 Maintenance of an inherited H3K27me3(-) chromatin state depends on antagonism of PRC2 by MES-4. a Diagram showing the cross between wildtype $P R C 2(+) K 27 m e 3(+)$ females and mes-3/mes-3 PRC2(-) K27me3(-) males that generates K27me3 M+P- F1 offspring. Maternal and paternal pronuclei are shown in the F1 1-cell embryo. The 2 primordial germ cells (PGCs) are shown in the newly hatched F1 larva. F1 hermaphrodites self-cross to produce F2 offspring. Figures that analyzed each generation/stage are indicated in parentheses. M, maternal; P, paternal; *Sensitized genetic background. Hybrid F1s were generated by mating Hawaii mothers with Bristol fathers. b Images of wild-type 2-cell embryos (left panels) or plk-1 2-cell embryos (right panels) demonstrate that the H3K27me3(-) state of sperm chromosomes in K27me3 $M+P$ - embryos (bottom panels) is easily monitored when sperm- and oocyteinherited chromosomes are kept in separate nuclei in plk-1 mutants. DAPI-stained DNA in red. H3K27me3 immunostaining in green. Scale bar represents $10 \mu \mathrm{m}$. c 2-cell and 8-cell stage $K 27 m e 3 M+P$ - embryos whose mothers were fed empty vector RNAi (control) or mes-4(RNAi) to knock down the maternal load of MES-4. DAPI-stained DNA in red. H3K27me3 immunostaining in green. Regions boxed in yellow in the left panels are shown at higher magnification in the right panels. Scale bar represents $10 \mu \mathrm{m}$. d Somatic cells and PGCs in a K27me3 M+P+ L1 (left) and a K27me3 M+P- L1 (right). L1 schematics show the observed staining pattern in PGCs for each genotype. DAPI-stained DNA in red. H3K27me3 immunostaining in green. Scale bar represents $2 \mu \mathrm{m}$ 
between inherited chromatin and offspring transcription requires that (1) genetically identical individuals display different transcriptional outcomes as a result of inheriting different chromatin states, (2) transcriptional changes must be demonstrated to occur in cis, thus eliminating alternative epigenetic carriers that function in trans (e.g., cytoplasmic ncRNAs), and (3) DNA methylation, which also functions in cis, must be eliminated as a potential mediator of the observed transcriptional changes. We met the third criterion by using the model system C. elegans, which lacks canonical DNA methylation. We met the remaining criteria by comparing transcription from sperm-inherited and oocyte-inherited alleles in genetically identical (hybrid fem-2/+; mes-3/+) worms (Fig. 1a) that inherited the sperm genome with or without $\mathrm{H} 3 \mathrm{~K} 27 \mathrm{me} 3$, which we call $K 27 m e 3 \mathrm{M}+\mathrm{P}+$ and K27me3 $M+P-$.

We showed previously that $K 27 m e 3 M+P$ - embryos have different chromatin states on their sperm-inherited and oocyteinherited genomes and that those states are maintained in all cells of early embryos ${ }^{12}$. As embryos develop, the gamete-inherited chromatin states are rewritten in somatic cells but maintained through the five cell divisions that generate the two primordial germ cells (PGCs) present in newly hatched larvae ${ }^{12}$ (Fig. 1d). When newly hatched larvae begin to feed, the PGCs launch their transcriptional program and begin to proliferate. During larval development, H3K27me3 gradually begins to accumulate on sperm-inherited chromosomes ${ }^{12}$. Absence of $\mathrm{H} 3 \mathrm{~K} 27 \mathrm{me} 3$ on sperm chromosomes at the onset of germ cell transcription could result in the activation of inappropriate genes. Once activated, a euchromatic state could be propagated at specific loci, sensitizing daughter germ cells to continued expression of inappropriate genes and potentially downstream target genes. We investigated whether global differences in inherited chromatin states in PGCs impact transcription in the developing germline. We used singlenucleotide polymorphisms (SNPs) between parental strains (from Bristol, England, and from Hawaii) to compare transcription from sperm-inherited and oocyte-inherited genomes. This allowed us to determine whether transcriptional changes occurred in cis (from sperm alleles specifically) or in trans (from both sperm and oocyte alleles).

Worms that inherited sperm chromosomes lacking H3K27me3 misexpressed genes in their germlines; 149 genes were upregulated and 116 genes were downregulated (FDR $<0.1)$ in $K 27 m e 3$ $\mathrm{M}+\mathrm{P}-$ compared to K27me3 $\mathrm{M}+\mathrm{P}+$ worms (Fig. 2a, Supplementary Figs. 2, 3). Of the genes that were upregulated in K27me3 $M+P$ - germlines, $96 \%$ are enriched for H3K27me3 in wild-type sperm $^{11}$ (Supplementary Fig. 4). While K27me3 $M+P$ - worms were typically fertile, they generated a significant proportion of sterile offspring (Fig. 2b). These findings demonstrate that altering sperm-inherited chromatin states results in changes to germline transcription and function.

Expression of genes assessed by reads overlapping SNPs between Bristol and Hawaii correlated well with expression assessed by using all reads mapping to a gene (Supplementary Fig. 3a). Therefore, we could use SNPs to determine, for the $23 \%$ of misexpressed genes that contain a SNP, if transcription differences arose from the sperm allele, the oocyte allele, or both (Supplementary Data 1). Our analysis of SNP-containing transcript reads indicated that downregulated genes reflect decreased transcription from both the sperm and oocyte allele. However, upregulated genes reflect increased transcription primarily from the sperm allele (Fig. 2c, d). Importantly, approximately half of these genes were upregulated in cis with increased transcription from the sperm allele specifically (dark red triangles in Fig. 2c). These findings demonstrate that differential marking on sperm-inherited and oocyte-inherited genomes allows sperm-inherited and oocyte-inherited alleles within the same cell to achieve different transcriptional outcomes in offspring. Given that C. elegans lacks canonical DNA methylation, this finding establishes a causal role for sperminherited $\mathrm{H} 3 \mathrm{~K} 27 \mathrm{me} 3$ in regulating transcription in offspring. It also raises the possibility that environmentally induced changes to gamete marking could lead to transcriptional changes in offspring, with developmental consequences.

The genes upregulated from both sperm and oocyte alleles likely represent downstream targets of an initially misexpressed factor(s). The observation that these genes are more upregulated from sperm alleles than oocyte alleles led us to hypothesize that the presence of $\mathrm{H} 3 \mathrm{~K} 27 \mathrm{me} 3$ on the oocyte genome acts as a barrier against transcriptional activation of germline-inappropriate genes. H3K27me3 on the oocyte-inherited genome would not be expected to protect it from factors that would shut down expression of germline-appropriate genes. This may explain the observation that downregulated genes were downregulated from both sperm and oocyte alleles. This led us to wonder if the global pattern of misexpression resulting from altered sperm chromatin was a concerted deviation from a germline program. To explore this, we performed gene ontology analysis on upregulated and downregulated genes. We found that downregulated genes are enriched for genes involved in reproduction and that upregulated genes are enriched for genes involved in somatic development, especially neuronal development (Supplementary Fig. 2b, c). This suggests that $K 27 m e 3 M+P-$ germlines are deviating from a germline program of gene expression toward a somatic program, perhaps favoring a neuronal program.

Sperm-inherited $\mathrm{H} 3 \mathrm{~K} 27 \mathrm{me} 3$ protects germ cell fate in offspring. To test whether germlines in $K 27 m e 3 \mathrm{M}+\mathrm{P}$ - worms that inherited altered sperm chromatin are in fact transitioning toward a neuronal fate, we analyzed worms carrying a panneuronal reporter gene, unc-119::GFP. We compared expression of this reporter in genetically identical worms that inherited sperm chromatin with either a wild-type pattern of $\mathrm{H} 3 \mathrm{~K} 27 \mathrm{me} 3$, $K 27 m e 3 M+P+$, or lacking $\mathrm{H} 3 \mathrm{~K} 27 \mathrm{me} 3, K 27 m e 3 M+P-$. As noted above, K27me3 $M+P-$ and $K 27 m e 3 M+P+$ worms are fertile; we observed that fertile germlines do not detectably express unc-119::GFP $(n>500)$ nor do they significantly upregulate $u n c-119$ transcript. Performing this analysis in a sensitized genetic background in which the maternal load of PRC2 was reduced by half (mes-3/+ heterozygous mother) rendered $\sim 30 \%$ of K27me3 $M+P-$ worms sterile $(n=267 / 894) ;<0.3 \%$ of $K 27 m e 3 M+P+$ worms were rendered sterile $(n=2 / 870)$. In this background, we found that the unc-119::GFP neuronal reporter was expressed in a significant percentage of sterile $K 27 m e 3 \mathrm{M}+P$ - adult germlines (Supplementary Table 1). Notably, expression of the neuronal reporter increased with age, from $16 \%$ in sterile day 1 adults to $31 \%$ in sterile day 2 adults. Expression of the neuronal reporter was rarely detected $(<0.1 \%)$ in germlines from late-stage larvae (L4) $(n=1 / 146)$ and never observed in the germlines from the rare sterile $K 27 m e 3 \mathrm{M}+\mathrm{P}+$ worms. These findings indicate that deviation from a germline program in $K 27 m e 3 \mathrm{M}+\mathrm{P}$ - worms is progressive and strongly suggest that loss of $\mathrm{H} 3 \mathrm{~K} 27 \mathrm{me} 3$ from the sperm-inherited genome results in a failure to maintain germ cell identity rather than a failure to establish it.

To test whether neuronal genes are derepressed from endogenous loci in $K 27 m e 3 M+P-$ germlines, we stained UNC-119::GFP(+) K27me3 $M+P$ - germlines for UNC-64, a neuronally expressed plasma membrane protein involved in synaptic vesicle fusion ${ }^{25}$. We detected this protein in all of the germlines analyzed $(n=6)$ (Fig. $3 c$ ). To test for loss of expression of genes involved in reproduction, we stained sterile $K 27 m e 3$ 
$K 27 m e 3 M+P-$ vs $M+P+$ mRNA reads in germlines (a,c,d)

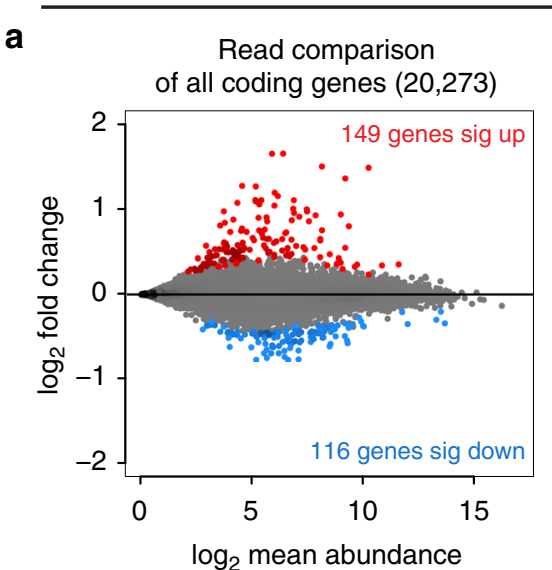

C

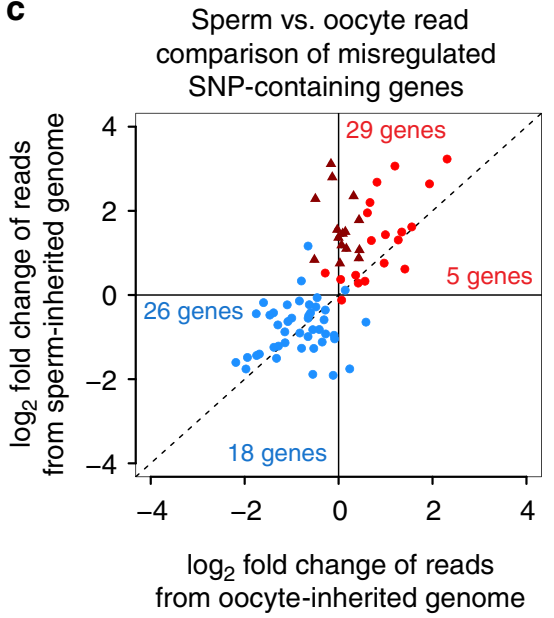

b
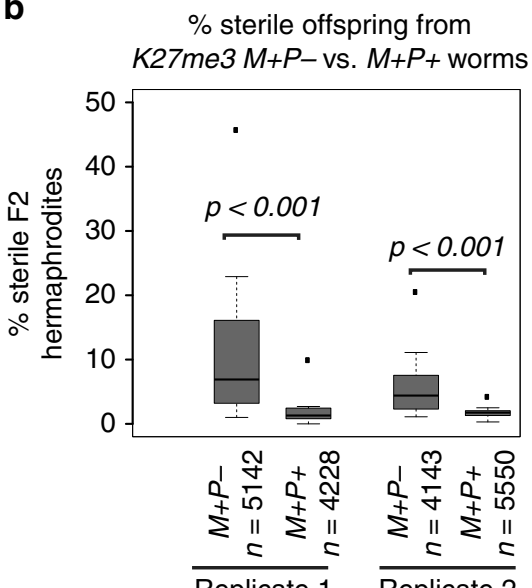

d
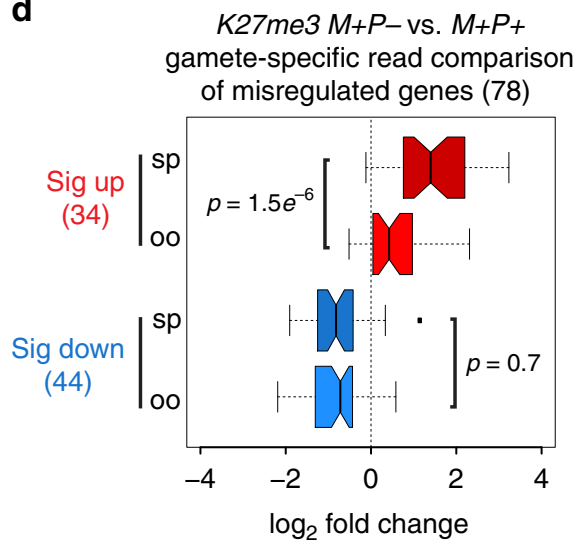

Fig. 2 Inheritance of sperm chromosomes lacking H3K27me3 alters germline transcription and function in offspring. a MA plot comparing germline transcripts (RNA-seq reads) from $K 27 m e 3 M+P$ - hermaphrodites to genetically identical $K 27 m e 3 M+P+$ hermaphrodites. Genes that are significantly upregulated or downregulated $(p<0.1)$ are highlighted in red and blue, respectively. $\mathbf{b}$ Boxplots showing percent sterility of the F2-generation hermaphrodites from K27me3 $M+P-$ and $K 27$ me3 $M+P+F 1$ worms for each of 2 replicates. $n=$ the number of F2 worms scored. Each box extends from the 25 th to the 75 th percentile, with the median indicated by the horizontal line; whiskers extend to the most extreme data point which is no more than 1.5 times the interquartile range from the box; individual data points outside of these boundaries are shown as filed boxes. $p$-values were calculated using a Wilcoxon rank sum test. c Scatter plot comparing sperm-specific and oocyte-specific $\log _{2}$ fold changes of SNP-containing reads from significantly upregulated genes (in red) and downregulated genes (in blue). Genes upregulated in cis ( $>1.5$-fold increase in reads from the sperm allele; $<1.5$-fold increase from the oocyte allele) are plotted as dark red triangles. The numbers of misregulated genes above and below the diagonal line are indicated. d Boxplots showing sperm-specific (sp) and oocyte-specific (oo) $\log _{2}$ fold changes of significantly upregulated genes (34 genes sig up) and significantly downregulated genes (44 sig down). Downregulated genes (blue) are similarly downregulated from sperm and oocyte alleles, while upregulated genes (red) are significantly more upregulated from sperm alleles than oocyte alleles (i.e., pairwise comparisons of transcript reads from sperm vs. oocyte alleles indicate that sperm allele reads are significantly more numerous than oocyte allele reads for upregulated genes but not for downregulated genes). Each box extends from the 25th to the 75th percentile, with the median indicated by the horizontal line; whiskers extend to the most extreme data point which is no more than 1.5 times the interquartile range from the box; the individual data point outside of these boundaries is shown as a filled box. The waist indicates the $95 \%$ confidence interval for the medians. $p$-values were generated using the paired student's $t$-test

$M+P-$ germlines for the germline proteins PGL-1 (a component of germ granules) ${ }^{26}$ and HTP-3 (a component of the synaptonemal complex $)^{27}$. We found that PGL-1 and HTP-3 were below detection (or nearly so) in $100 \%(n=27)$ and $28 \%(n=60)$ of sterile $K 27 m e 3 M+P$ - germlines analyzed, respectively, regardless of whether or not they expressed the neuronal GFP reporter (Fig. 3d, e). Although only $28 \%$ of sterile K27me3 $\mathrm{M}+\mathrm{P}-$ germlines lacked detectable HTP-3 staining, the remaining $72 \%$ of germlines analyzed displayed abnormal HTP-3 patterns (Fig. 3d). Of note, germlines expressing the neuronal UNC119::GFP reporter consistently displayed more severe HTP-3 phenotypes than germlines that were UNC-119::GFP(-) (Fig. 3d).
Consistent with $K 27 m e 3 M+P$ - germlines adopting a neuronal identity, we readily observed patches of highly concentrated GFP signal in UNC-119::GFP $(+)$ germlines, indicating that the normally syncytial germ cells had cellularized. In some cases, we observed axo-dendritic structures emanating from these bright $\mathrm{GFP}(+)$ cells (Fig. 3b, c). Our findings demonstrate that absence of $\mathrm{H} 3 \mathrm{~K} 27 \mathrm{me} 3$ from the sperm-inherited genome results in the derepression of many somatic genes, especially neuronal genes, predominantly from sperm-inherited alleles. In a sensitized background, lack of $\mathrm{H} 3 \mathrm{~K} 27 \mathrm{me} 3$ on the sperm-inherited genome results in germlines that transition away from a germline program and toward a neuronal program. 
Sensitized $K 27 m e 3 M+P$ - sterile worms
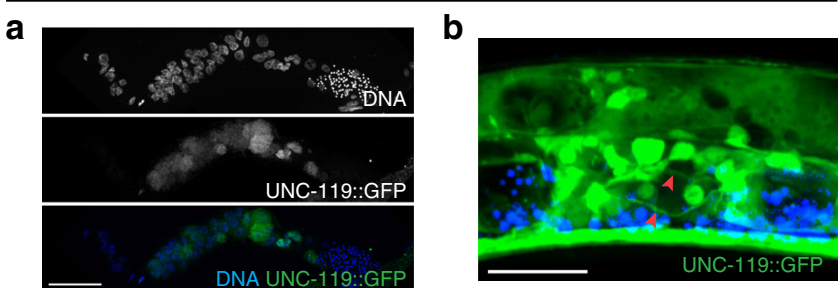

C

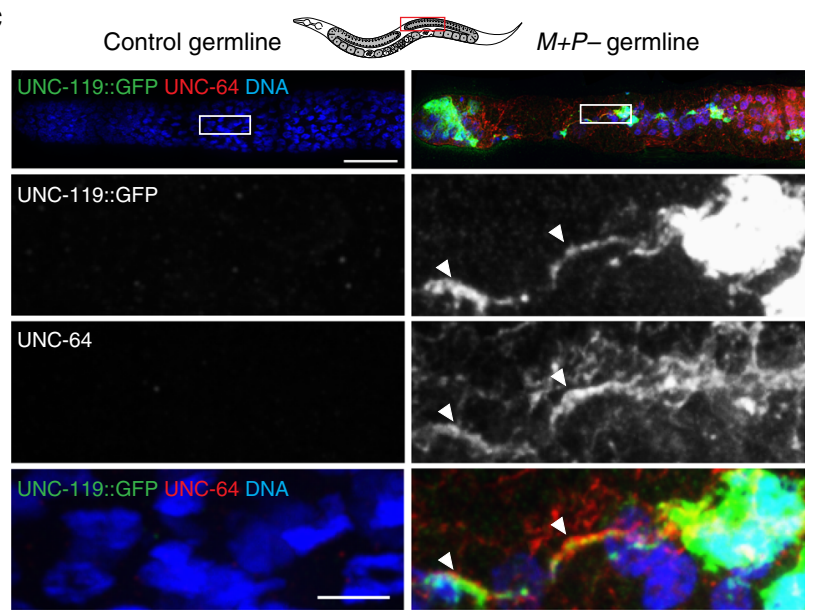

d

Percent of control and sterile $K 27 m e 3 M+P$ - worms with different patterns of germline-specific protein HTP-3

\begin{tabular}{|c|c|c|c|c|}
\hline & Chromosomal & $\begin{array}{c}\text { Reduced } \\
\text { chromosomal }\end{array}$ & $\begin{array}{c}\text { Not } \\
\text { chromosomal }\end{array}$ & $\begin{array}{c}\text { Nearly } \\
\text { below detection }\end{array}$ \\
\hline $\begin{array}{c}\text { Control } \\
(n=31)\end{array}$ & $100 \%$ & - & - & - \\
\hline $\begin{array}{c}\text { M+P- } \\
\text { GFP(-l+) } \\
(n=60)\end{array}$ & - & $22 \%$ & $50 \%$ & $28 \%$ \\
$\begin{array}{c}\text { GFP(-) } \\
(n=31)\end{array}$ & - & $42 \%$ & $35 \%$ & $23 \%$ \\
\hline $\begin{array}{c}\text { GFP }(+) \\
(n=29)\end{array}$ & - & - & $66 \%$ & $34 \%$ \\
\hline $\begin{array}{c}\text { Pachytene } \\
\text { germline } \\
\text { nuclei }\end{array}$ & HTP-3 & DNA & & \\
\hline
\end{tabular}

$\mathbf{e}$

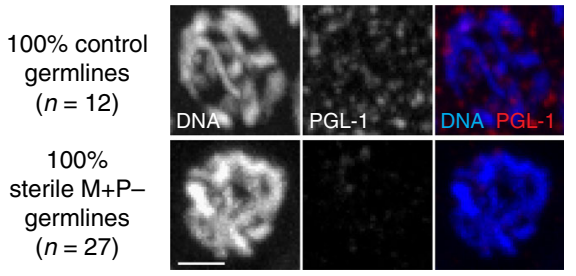

\section{Discussion}

Establishment and maintenance of chromatin states are critical during development and aging. During early development, inherited chromatin states must be rewritten to enable differentiation of different lineages. During aging, established chromatin states must be maintained to preserve cell-type-specific gene expression patterns and thus protect cell identity. Defects in these processes can lead to severe developmental defects and
Fig. 3 Inheritance of sperm chromosomes lacking H3K27me3 causes germ cells in offspring to transition toward a neuronal fate. a Immunofluorescence images of a sterile germline from a K27me3 $M+P-$ worm in a sensitized genetic background (see text) and carrying an unc-119::gfp neuronal reporter transgene. DNA in blue. GFP in green. Scale bar represents $40 \mu \mathrm{m}$. b Image of GFP in a live K27me3 $M+P$ - worm carrying the unc-119::gfp reporter transgene. Arrowheads point to axo-dendritic processes extending from cells in the gonad with high levels of UNC-119::GFP. GFP in green. Blue is autofluorescent gut granules. Scale bar represents $10 \mu \mathrm{m}$. c Immunofluorescence images of a control germline and a sterile germline from a K27me3 $\mathrm{M}+\mathrm{P}$ - worm showing expression of the UNC-119::GFP reporter in green, immunostaining of the neuronally expressed protein UNC64 in red, and DNA in blue. Worm schematic indicates the region of germline images. Arrowheads point to axo-dendritic processes co-stained for UNC-119::GFP and UNC-64 in the zoomed-in images. Scale bar in top panel represents $30 \mu \mathrm{m}$. Scale bar in lower panel represents $5 \mu \mathrm{m}$. d, e Summary of HTP-3 staining (d) and PGL-1 staining (e) observed in control germlines from fertile K27me3 $M+Z-$; unc-119:::fp worms and germlines from sterile $K 27 m e 3 M+P-$ worms. For each staining category, representative immunofluorescence images of DNA and HTP-3 or PGL-1 are shown. Scale bar represents $10 \mu \mathrm{m}$ (d) and $2 \mu \mathrm{m}$ (e)

cancer. Whether chromatin states established in the parent and transmitted to offspring via sperm or oocyte influence the establishment and/or maintenance of cell fates in those offspring is a critical question in the field. Our findings demonstrate that in C. elegans sperm-inherited chromatin states influence germ cell identity in offspring. Because we observe reprogramming of germ cells toward neuronal cells in adult $K 27 \mathrm{me} 3 \mathrm{M}+P$ - germlines but only rarely in larval $K 27 \mathrm{me} 3 \mathrm{M}+P$ - germlines, we conclude that offspring germ cells lose rather than fail to establish their germ cell identity. These findings reveal that in worms, sperm-inherited H3K27me3 is transmitted to offspring germ cells to prevent germline-inappropriate transcription and thus protect germ cell identity.

Altogether, our findings point to a model in which gameteinherited patterns of $\mathrm{H} 3 \mathrm{~K} 27 \mathrm{me} 3$ act as a barrier to inappropriate transcription; in germ cells, this barrier function is particularly important in suppressing transcription of factors that could drive germ cells toward alternative cell identities (Fig. 4). In the germlines of worms that inherited the sperm genome lacking H3K27me3, we speculate that some genes become upregulated as a direct result of diminished H3K27me3 (Factor X in Fig. 4), while other genes may be upregulated as a consequence of being the target of an upregulated gene. An alternative scenario is that Factor X genes are normally expressed in wild-type germ cells but prevented from activating germline-inappropriate target genes by H3K27me3 on those target genes. An example of the first scenario (Fig. 4 ) is the vab-7 gene, which encodes a neuronal transcription factor ${ }^{28}$; $v a b-7$ is not expressed in wild-type germlines but becomes upregulated in K27me3 $M+P-$ germlines. Examples of the alternative scenario are genes that encode neuronal specification factors (e.g., die- 1 and $n s y-7)^{28}$ that are highly expressed in both wild-type and K27me3 $M+P$ - germlines. Regardless of the exact cascade of gene activation, our expression analysis of K27me3 $M+P$ - germlines suggests a programmatic shift toward a neuronal program and supports the hypothesis that an upstream factor(s) is altering gene expression in a concerted manner, especially from the H3K27me3-depleted sperm genome.

Our findings establish a causal role for sperm-inherited histone marks influencing transcription in offspring and reveal their role in protecting cell identity. Together with observations that in mammals, sperm retain modified histones over developmentally important genes ${ }^{5-7}$, this work implicates sperm-inherited chromatin states as one mechanism through which environmentally 


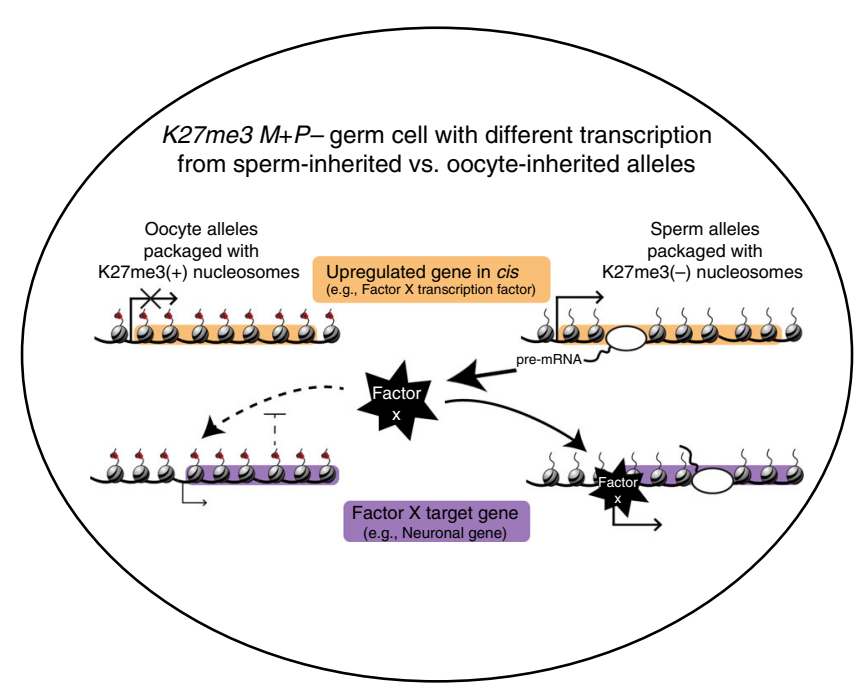

Fig. $4 \mathrm{~A}$ model for transcriptional upregulation in germ cells that inherited sperm chromosomes lacking H3K27me3. The schematic is a germ cell nucleus from a $K 27 m e 3 \mathrm{M}+\mathrm{P}$ - worm showing oocyte-inherited and sperminherited alleles for 2 representative genes: a transcription factor-encoding gene (orange) and a target gene of that transcription factor (purple). Sperm-inherited alleles lack repressive H3K27me3 and as a result are prone to upregulate Transcription Factor X. If expressed, Transcription Factor $X$ can then activate target genes strongly from H3K27me3(-) sperm alleles and weakly from H3K27me3(+) oocyte alleles. In this model, H3K27me3 would act as a barrier against (1) production of Factor $X$ and (2) activation of Factor $\mathrm{X}$ target genes. In K27me3 $\mathrm{M}+\mathrm{P}+$ worms, oocyte-inherited and sperm-inherited alleles are similarly marked with $\mathrm{H} 3 \mathrm{~K} 27 \mathrm{me} 3$

induced changes to parental epigenomes can impact the development and health of future generations.

\section{Methods}

C. elegans growth conditions. Worms were maintained on nematode growth medium (NGM) agar plates spotted with Escherichia coli OP50 and maintained at $15^{\circ} \mathrm{C}$ (fem-2 and plk-1 containing strains) or $20^{\circ} \mathrm{C}$ (all other strains). To generate females, fem-2 strains were raised at $24^{\circ} \mathrm{C}$ until L4.

\section{C. elegans strains. All strains are in the N2 background except CB4856 and} SS1292.

N2 wild-type isolate from Bristol, England.

CB4856 wild-type isolate from Hawaii.

SS1292 fem-2(b245ts) III - mutation CRISPR-engineered into CB4856.

DH0245 fem-2(b245ts) III

SS1229 fem-2(b245ts) III; mes-4(bn73) $V$

SS1167 mes-3(bn35) I/hT2-GFP (I;III); fem-2(b245ts) III.

SS1099 mes-3(bn35) I/hT2-GFP (I;III); him-8(e1489) IV.

OCF65 plk-1(or683) III; fog-1(e2121) unc-11(e47) I/hT2 [qIs48] (I:III); itIs37

[pAA64: pie-1p::mCHERRY::his-58+unc-119(+)].

SS1424 mes-3(bn35) I/hT2-GFP(I;III); edIs6[unc-119::GFP+rol-6(su1006)] IV.

SS1298 set-2(bn129) fem-2(b245ts) III.

Fixing and staining embryos, larvae, and germlines. Specimens were flattened between a slide and a coverslip by wicking away buffer (see below). Slides were frozen in liquid nitrogen, coverslips were removed, then slides were fixed in methanol $(10 \mathrm{~min})$ and acetone $(10 \mathrm{~min})$ and allowed to air dry for up to 3 days. Slides were blocked ( $1.5 \%$ ovalbumin, $1.5 \%$ BSA in PBS) for 25 min, then incubated with primary antibodies overnight at $4{ }^{\circ} \mathrm{C}$. Slides were washed $3 \times$ in PBST $(0.1 \%$ tween in phosphate buffered saline $\mathrm{pH}$ 7.2), blocked for $5 \mathrm{~min}$, incubated with secondary antibodies and DAPI at room temperature for $2 \mathrm{~h}$, and washed $3 \times$ in PBST. Coverslips were mounted on slides with gelutol. Slides were allowed to dry for up to 3 days before being imaged.

Antibodies. Primary antibodies and their dilutions were as follows: 1:30,000 of $2.45 \mathrm{mg} / \mathrm{mL}$ mouse anti-H3K27me3 (Kimura mAb 1E7 clone CMA323, Wako cat\#309-95259), 1:3000 original stock solution of rabbit anti-H3K27me3 (C36B11 Cell Signaling MAb\#9733 lot\#C36B11), 1:4000 of $0.4 \mathrm{mg} / \mathrm{mL}$ mouse anti-GFP (Roche cat\#11 814460001 lot\#14158300), 1:4000 rabbit anti-UNC-64 serum²2,
1:50,000 rabbit anti-PGL-1 serum ${ }^{23}, 1: 500$ guinea pig anti-HTP-3 serum ${ }^{24}$. Secondary antibodies conjugated to Alexa Fluor: 488 goat anti-mouse (Life cat\#A11001), 488 goat anti-rabbit (Molecular Probes cat\#A-11008), 594 goat antirabbit (Life cat\#A11012), and 594 goat anti-guinea pig (Molecular Probes cat\#A11076) were used at 1:300 with $0.05 \mu \mathrm{g} / \mathrm{mL}$ 4',6-diamidino-2-phenylindole (DAPI).

Microscope set-up for immunocytochemistry. All images were acquired using the following: Yokogawa CSUX-1 spinning disk scanner, Nikon (Garden City, NY) TE2000-E inverted stand, Hamamatsu ImageEM X2 camera, solid state 405, 488, and $561 \mathrm{~nm}$ laser lines, 435-485, 500-550, and 573-613 fluorescent filters, Nikon Plan Apo VC 60×/1.40 oil objective, Nikon Plan Apo 100×/1.40 oil objective, and Micro-Manager software.

Analysis of MES-4-depleted and SET-2 mutant embryos. plk-1; fog-1 unc-11 females (Fig. 1b, c) or fem-2(ts) or set-2 fem-2(ts) females (Supplementary Fig. 1) were mated to $\mathrm{N} 2(\mathrm{P}+)$ or mes-3 M-Z-; him- $8(\mathrm{P}-)$ males ( $\mathrm{M}$ - for no maternal load of MES-3, Z- for no zygotic synthesis of MES-3) on plates with mes-4 RNAi, empty vector RNAi, or OP50 (Supplementary Fig. 1c) and incubated overnight at $23^{\circ} \mathrm{C}$ (Fig. 1b, c) or $20^{\circ} \mathrm{C}$ (Supplementary Fig. 1). Embryos were dissected from mated females in egg buffer ( $25 \mathrm{mM}$ HEPES pH 7.4, $118 \mathrm{mM} \mathrm{NaCl}, 48 \mathrm{mM} \mathrm{KCl}, 2 \mathrm{mM}$ $\mathrm{CaCl}_{2}, 2 \mathrm{mM} \mathrm{MgCl}$ ) with $1 \mathrm{mM}$ levamisole on polylysine-coated slides. Embryos attached to slides were fixed and stained as described above. Stacks of optical sections were acquired on a Solamere spinning disk confocal system as described above. Stacks were collapsed into maximum intensity projections using MicroManager. Single-channel and merge images were generated in Photoshop. To assess the appearance of $\mathrm{H} 3 \mathrm{~K} 27 \mathrm{me} 3$ on sperm chromosomes in embryos with and without a maternal load of MES-4, optical sections of K27me3 $M+P$ - embryos from $f e m-2(t s)$ mothers (with a maternal load of MES-4) or from fem-2(ts); mes-4(bn73) mothers (lacking a maternal load of MES-4) were stained for DNA and H3K27me3 and scored for: 1) undetectable H3K27me3 on some chromosomes, 2) reduced levels of $\mathrm{H} 3 \mathrm{~K} 27 \mathrm{me} 3$ on some chromosomes relative to other chromosomes, or 3) indistinguishable levels of $\mathrm{H} 3 \mathrm{~K} 27 \mathrm{me} 3$ on all chromosomes.

Analysis of $\mathbf{K 2 7 m e 3} \boldsymbol{M}+\boldsymbol{P}-$ and $\boldsymbol{M}+\boldsymbol{P}+\mathbf{L} \mathbf{1}$ larvae. fem- $2(t s)$ females were mated to N2 $(\mathrm{P}+)$ or mes-3 M-Z-; him-8 $(\mathrm{P}-)$ males for $\sim 48 \mathrm{~h}$. Gravid females were transferred to $100 \mu \mathrm{L}$ S Basal $\left(5.85 \mathrm{~g} \mathrm{NaCl}, 1 \mathrm{~g} \mathrm{~K}_{2} \mathrm{HPO}_{4}, 6 \mathrm{~g} \mathrm{KH}_{2} \mathrm{PO}_{4}, 1 \mathrm{~mL}\right.$ cholesterol $\left(5 \mathrm{mg} / \mathrm{mL}\right.$ in ethanol), $\mathrm{H}_{2} 0$ to $1 \mathrm{~L}$ ) on gelatin chrom alum-coated slides and incubated overnight in a humid chamber at $20^{\circ} \mathrm{C}$. Starved K27me3 $M+P-$ and K27me3 $M+P+$ L1s were transferred to polylysine-coated slides. L1s attached to slides were fixed and stained as described above. Stacks of optical sections were acquired on a Solamere spinning disk confocal system as described above. Stacks were collapsed into maximum intensity projections using Micro-Manager. Singlechannel and merge images were generated in Photoshop.

Analysis of sensitized $\mathbf{K 2 7 m e} \mathbf{M}+\boldsymbol{P}-$ and $\boldsymbol{M}+\boldsymbol{P}+$ germlines. mes-3/hT2-GFP, fem-2(ts) females were mated to either mes-3/hT2-GFP; unc-119 (P+) or mes-3 M $+Z-$; unc-119 $(\mathrm{P}-)$ males to generate K27me3 $M+P+$ and K27me3 $M+P-$ worms, respectively. $146 \mathrm{~L} 4 \mathrm{~K} 27 \mathrm{me} 3 \mathrm{M}+P$ - larvae were cut at the pharynx to extrude their anterior germline for assessment of UNC-119::GFP expression in germ cells. Sterile adults on day 1 of adulthood $(n=267)$ were moved to new plates for live analysis to determine whether germ cells expressed UNC-119::GFP. Sterile day 1 adults that lacked germline expression of UNC-119::GFP were allowed to age at $20^{\circ} \mathrm{C}$ for a repeat analysis of UNC-119::GFP expression in germ cells on day 2 of adulthood. L4s, day 1 sterile adults, and day 2 sterile adults were analyzed for germ cell expression of UNC-119::GFP on a Leica MZ16F microscope equipped with a GFP filter. Germlines were imaged for GFP expression both by live imaging of whole worms and by fixing dissected germlines in egg buffer with $1 \mathrm{mM}$ levamisole and $0.5 \%$ tween on polylysine-coated slides and immunostaining for GFP, UNC64, PGL-1, and/or HTP-3. Stacks of optical sections were acquired on a Solamere spinning disk confocal system controlled by Micro-Manager software as described above. Stacks were collapsed into maximum intensity projections using MicroManager and stitched together into composite images using Photoshop. Singlechannel and merge images were generated in Photoshop. In some cases (Fig. 3c), germlines were straightened using ImageJ.

Sterility analysis of $\boldsymbol{K} 27 \mathrm{me} \mathbf{3} \boldsymbol{M}+\boldsymbol{P}-$ and $\boldsymbol{M}+\boldsymbol{P}+$ offspring. fem- $2(t s)$ females were mated to mes-3/hT2-GFP; fem-2 $(\mathrm{P}+)$ or mes-3 $M+Z-$; fem- $2(\mathrm{P}-)$ males at $15^{\circ} \mathrm{C}$. mes-3/+; fem-2 K27me3 $M+P+$ and $M+P-$ L4s $(n=30$ replicate $1 ; n=40$ replicate 2) were cloned to individual plates and allowed to lay eggs for $\sim 24 \mathrm{~h}$, then transferred to fresh plates each day until no more embryos were laid. Embryos from $K 27 m e 3 M+P+$ and $M+P$ - hermaphrodites were incubated at $15^{\circ} \mathrm{C}$ until day 1 adulthood, then hermaphrodites were scored as sterile (no embryos in the uterus) or fertile ( $n=9370$ replicate $1 ; n=9693$ replicate 2$)$.

RNA-seq of $K 27 m e 3 \mathbf{M}+\boldsymbol{P}-$ and $\boldsymbol{M}+\boldsymbol{P}+$ hybrid and non-hybrid germlines Feminized fem-2 (CB4856 (hybrid) or N2 (non-hybrid) background) hermaphrodites were mated to either mes-3/hT2-GFP; him-8 $(\mathrm{P}+)$ or mes-3 M-Z-; him-8 
males $(\mathrm{P}-)$ to generate genetically identical mes-3/+; fem-2/+; him-8/+ K27me3 $M+P+$ and $M+P-$ offspring, respectively. In addition, feminized fem-2 (CB4856 background) were mated to wild-type N2 males to generate "wild-type" hybrid offspring. mes-3/+; fem-2/+; him-8/+(K27me3 $M+P+$ and $M+P-)$ and fem-2/+ ("wild-type" hybrid) L4 offspring were picked and allowed to age overnight to generate K27me3 $M+P+$, K27me3 $M+P-$, and "wild-type" hybrid day 1 adults. $\sim 50$ germlines were dissected from day 1 adults in egg buffer with $1 \mathrm{mM}$ levamisole, $0.5 \%$ tween and transferred into $300 \mu \mathrm{L}$ of ice-cold TRIzol reagent (Life Technologies), then stored at $-80^{\circ} \mathrm{C}$. Samples were subjected to 3 freeze-thaw cycles. One microliter linear polyacrylamide was added to each sample, vortexed, then transferred to Phase Lock Gel-Heavy $2 \mathrm{~mL}$ tubes to extract the aqueous phase. 0.75 volume isopropanol was added to each sample and incubated overnight at $-20^{\circ} \mathrm{C}$. RNA was isolated via ethanol precipitation and rehydrated in $14.5 \mu \mathrm{L}$ RNase-free $\mathrm{H}_{2} \mathrm{O}$. 2.5 $\mu \mathrm{L}$ were used to assess RNA quality (Agilent RNA Nano Bioanalyzer Chip) and concentration (Qubit Quant-IT RNA Assay Kit). The remaining $12 \mu \mathrm{L}$ were depleted of rRNA (NEBNext rRNA Depletion Kit) and used for library preparation (NEBNext Ultra RNA Library Prep Kit). Libraries were sequenced on an Illumina HiSeq4000 to acquire paired-end $100 \mathrm{bp}$ reads. Four biological replicates each were generated for K27me3 $M+P-$, K27me3 $M+P+$, and "wild-type" hybrid samples.

Processing and analyzing RNA-seq data. For differential expression analysis, raw sequences were mapped to transcriptome version WS220 using TopHat 22 . Only reads with one unique mapping were allowed. Otherwise default options were used. Reads mapping to ribosomal RNAs were removed from further analysis. HTSeq ${ }^{30}$ was used to obtain read counts per transcript. DESeq $2^{31}$ was used to determine differentially expressed genes from HTSeq counts. A Benjamini-Hochberg multiple hypothesis corrected $p$-value cutoff of 0.1 was used as a significance cutoff to identify significantly differentially expressed genes between $K 27 m e 3 \mathrm{M}+\mathrm{P}-$ and $M+P+$ germlines.

To identify RNA-sequencing (RNA-seq) reads coming from the Hawaiian or Bristol parent in the hybrid offspring germlines, the annotated single nucleotide polymorphisms (SNPs) of the Hawaiian strain CB4856 genome compared to the Bristol strain N2 WS220 genome were downloaded from WormBase. 126,615 unique SNPs were downloaded, of which 25,980 SNPs mapped to an exon in a total of 9146 coding genes, according to WormBase. Henceforth, we refer to these SNPs as exonic SNPs.

RNA-seq reads from the Bristol strain, Hawaiian strain, and hybrids were mapped to the Bristol (N2) genome version WS220 using TopHat2, once allowing 1 mismatch in a read and a second time not allowing a mismatch. Reads overlapping exonic SNP positions were identified, and HTSeq was used to count these SNP-containing reads for each transcript. Counts per transcript were normalized by the number of total transcript reads, including non-SNPoverlapping reads, for each of the libraries sequenced. An average count per transcript was obtained by averaging the replicates for each of the Bristol, Hawaiian, and hybrid $K 27 m e 3 M+P-$ and $M+P+$ replicate experiments. A further quality control step was imposed. For the Bristol and Hawaiian RNA-seq data, we calculated a distance score between the mismatch 0 and mismatch 1 read counts for each transcript. For each transcript $\mathrm{i}$ in the Hawaiian RNA-seq data, a score $\mathrm{h} \mathrm{z}_{\mathrm{i}}$ was

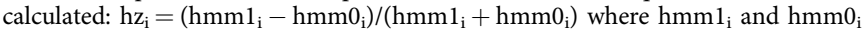
denote the normalized read counts for transcript i from mapping with a mismatch 1 and with a mismatch 0 to the Bristol genome, respectively. In the same way, we defined $b z_{i}, b m m l_{i}$, and $b m m 0_{i}$ for transcripts from mapping our Bristol RNA-seq data. For Hawaiian transcript reads from exonic SNPs, we expect $h z_{i}$ to be close to 1; all reads should have mapped when allowing 1 mismatch, and none should have mapped when allowing no mismatches. For Bristol transcript reads from exonic SNPs, we expect $b z_{i}$ to be close to 0 , as the number of reads from both mappings should have been very similar. We removed transcripts that did not

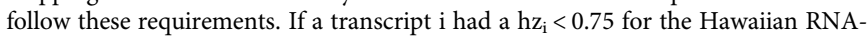
seq data, or an absolute value $\left|\mathrm{bz}_{\mathrm{i}}\right|>0.25$ for the Bristol RNA-seq data, that transcript was removed from further analysis. These requirements removed 1080 transcripts with exonic SNPs. For the remaining 8066 transcripts we obtained from the hybrid $K 27 m e 3 M+P-$ and $M+P+$ RNA-seq data, we determined the transcript reads from the sperm allele (Bristol) as reads that mapped when allowing 0 mismatches. We determined the transcript reads from the oocyte allele (Hawaii) as reads that mapped when allowing 1 mismatch but that did not map when allowing 0 mismatches. We thus calculated transcript reads from the oocyte allele by subtracting reads that mapped when allowing 0 mismatches (sperm allele reads) from reads that mapped when allowing 1 mismatch (reads from both gamete alleles).

Many genes with exonic SNPs were not expressed in any of our germline RNAseq data. We filtered out these non-expressed transcripts from further analysis by requiring the normalized read counts from the sperm allele or oocyte allele in at least one condition, $K 27 m e 3 M+P-$ or $M+P+$, to be at least 0.5 . Because our goal was to use SNP-containing transcript reads as a proxy for total transcript reads, we filtered out transcripts that had a greater than 2.5-fold difference between the $\log _{2}$ of SNP-containing reads versus the $\log _{2}$ of total reads for a given transcript in hybrid germlines (Hawaii mother and Bristol father). This requirement removed 190 genes and left 2,606 transcripts for further analysis. To avoid dividing by zero when calculating log fold changes and to reduce the effect of noise on low read counts, we added a pseudo-count of 0.5 to all remaining transcripts from oocyte alleles and sperm alleles in both conditions.

GO analysis of significantly misregulated genes. Significantly upregulated genes (361 genes) and downregulated genes (342 genes) in the "wild-type" hybrid vs $K 27 m e 3 M+P-$ comparison $(\mathrm{FDR}<0.05)$ were used to perform $\mathrm{GO}$ analysis (Supplementary Fig. 2b, c). GO analysis was performed using the tissue enrichment tool provided by WormBase (version WS266).

Statistics. Sample sizes and statistical tests used are described in the figure legends. Paired student's $t$-tests and Wilcoxon rank sum tests were performed using R. Four biological replicates of sequencing data were used. No replicates were omitted. Differential expression analysis was performed using DESeq2, which uses negative binomial generalized linear models. Adjusted $p$-values were calculated using the Benjamini-Hochberg method performed by DESeq2.

Reporting Summary. Further information on experimental design is available in the Nature Research Reporting Summary linked to this article.

\section{Data availability}

Raw sequence files and processed data sets generated in this study are available at the Gene Expression Omnibus (GEO) repository, accession GSE123415. Processed data sets available at GEO are as follows: RNA-seq read counts for K27me3 $M+P-, K 27 m e 3 M+P$ + and "wild-type" hybrid containing (1) all read counts mapping to a transcript, (2) read counts overlapping SNPs mapped to the Bristol genome allowing 1 mismatch (Hawaii \& Bristol reads), (3) read counts overlapping SNPs mapped to the Bristol genome allowing 0 mismatches (Bristol-specific reads), (4) differential expression analysis comparing transcript reads from $K 27 m e 3 M+P-$ vs. $M+P+$, and (5) differential expression analysis comparing transcript reads from $K 27 m e 3 M+P$ - vs. "wild-type" hybrid. Supplementary Data 1 consists of sperm-specific and oocyte-specific reads and the $\log _{2}$ fold changes between $K 27 m e 3 M+P-$ and $M+P+$ for significantly misregulated SNP-containing genes. All other relevant data supporting the key findings of this study are available within the article and its Supplementary Information files or from the corresponding author upon reasonable request. A reporting summary for this Article is available as a Supplementary Information file.

Received: 19 November 2018 Accepted: 21 February 2019

Published online: 20 March 2019

\section{References}

1. Pembrey, M., Saffery, R. \& Bygren, L. O. Human transgenerational responses to early-life experience: potential impact on development, health and biomedical research. J. Med. Genet. 51, 563-72 (2014).

2. Vågerö, D., Pinger, P. R., Aronsson, V. \& van den Berg, G. J. Paternal grandfather's access to food predicts all-cause and cancer mortality in grandsons. Nat. Commun. 9, 5124 (2018).

3. Adalsteinsson, B. T. \& Ferguson-Smith, A. C. Epigenetic control of the genome-lessons from genomic imprinting. Genes 5, 635-655 (2014).

4. Gapp, K. \& Bohacek, J. Epigenetic germline inheritance in mammals: looking to the past to understand the future. Genes Brain Behav. 17, e12407 (2018).

5. Yamaguchi, K. et al. Re-evaluating the localization of sperm-retained histones revealed the modification-dependent accumulation in specific genome regions. Cell Rep. 23, 3920-3932 (2018).

6. Yoshida, K. et al. Mapping of histone-binding sites in histone replacementcompleted spermatozoa. Nat. Commun. 9, 3885 (2018).

7. Jung, Y. H. et al. Chromatin states in mouse sperm correlate with embryonic and adult regulatory landscapes. Cell Rep. 18, 1366-1382 (2017).

8. Teperek, M. et al. Sperm is epigenetically programmed to regulate gene transcription in embryos. Genome Res. 26, 1034-1046 (2016).

9. Siklenka, K. et al. Disruption of histone methylation in developing sperm impairs offspring health transgenerationally. Science 350, aab2006 (2015).

10. Klosin, A., Casas, E., Hidalgo-Carcedo, C., Vavouri, T. \& Lehner, B. Transgenerational transmission of environmental information in C. elegans. Science 356, 320-323 (2017).

11. Tabuchi, T. M. et al. Caenorhabditis elegans sperm carry a histone-based epigenetic memory of both spermatogenesis and oogenesis. Nat. Commun. 9, 4310 (2018).

12. Gaydos, L. J., Wang, W. \& Strome, S. H3K27me and PRC2 transmit a memory of repression across generations and during development. Science 345, 1515-1518 (2014)

13. Rechtsteiner, A. et al. The histone H3K36 methyltransferase MES-4 acts epigenetically to transmit the memory of germline gene expression to progeny. PLoS Genet. 6, e1001091 (2010). 
14. Gaydos, L. J., Rechtsteiner, A., Egelhofer, T. A., Carroll, C. R. \& Strome, S. Antagonism between MES-4 and polycomb repressive complex 2 promotes appropriate gene expression in C. elegans germ cells. Cell Rep. 2, 1169-1177 (2012).

15. Capowski, E. E., Martin, P., Garvin, C. \& Strome, S. Identification of grandchildless loci whose products are required for normal germ-line development in the nematode Caenorhabditis elegans. Genetics 129 1061-1072 (1991).

16. Margueron, R. et al. Role of the polycomb protein EED in the propagation of repressive histone marks. Nature 461, 762-767 (2009).

17. Poepsel, S., Kasinath, V. \& Nogales, E. Cryo-EM structures of PRC2 simultaneously engaged with two functionally distinct nucleosomes. Nat. Struct. Mol. Biol. 25, 154-162 (2018).

18. Yuan, W. et al. H3K36 methylation antagonizes PRC2-mediated H3K27 methylation. J. Biol. Chem. 286, 7983-7989 (2011).

19. Schmitges, F. W. et al. Histone methylation by PRC2 is inhibited by active chromatin marks. Mol. Cell 42, 330-341 (2011).

20. Bender, L. B. et al. MES-4: an autosome-associated histone methyltransferase that participates in silencing the $\mathrm{X}$ chromosomes in the C. elegans germ line. Development 133, 3907-3917 (2006).

21. Xiao, Y. et al. Caenorhabditis elegans chromatin-associated proteins SET-2 and ASH-2 are differentially required for histone H3 Lys 4 methylation in embryos and adult germ cells. Proc. Natl Acad. Sci. 108, 8305-8310 (2011).

22. Rahman, M. M. et al. Caenorhabditis elegans polo-like kinase PLK-1 is required for merging parental genomes into a single nucleus. Mol. Biol. Cell 26, 4718-4735 (2015).

23. Kreher, J. et al. Distinct roles of two histone methyltransferases in transmitting H3K36me3-based epigenetic memory across generations in Caenorhabditis elegans. Genetics 210, 969-982 (2018).

24. Højfeldt, J. W. et al. Accurate H3K27 methylation can be established de novo by SUZ12-directed PRC2. Nat. Struct. Mol. Biol. 25, 225-232 (2018).

25. Saifee, O., Wei, L. \& Nonet, M. L. The Caenorhabditis elegans unc-64 locus encodes a syntaxin that interacts genetically with synaptobrevin. Mol. Biol. Cell 9, 1235-1252 (1998).

26. Kawasaki, I. et al. PGL-1, a predicted RNA-binding component of germ granules, is essential for fertility in C. elegans. Cell 94, 635-645 (1998).

27. MacQueen, A. J. et al. Chromosome sites play dual roles to establish homologous synapsis during meiosis in C. elegans. Cell 123, 1037-1050 (2005).

28. Hobert, O. Patterning and Cell Type Specification in the Developing CNS and PNS. 609-626 (Elsevier, London, 2013).

29. Kim, D. et al. TopHat2: accurate alignment of transcriptomes in the presence of insertions, deletions and gene fusions. Genome Biol. 14, R36 (2013).

30. Anders, S., Pyl, P. T. \& Huber, W. HTSeq-a Python framework to work with high-throughput sequencing data. Bioinformatics 31, 166-169 (2015).

31. Love, M. I., Huber, W. \& Anders, S. Moderated estimation of fold change and dispersion for RNA-seq data with DESeq2. Genome Biol. 15, 550 (2014).

\section{Acknowledgements}

We thank Abby Dernburg for HTP-3 antibody, Ben Abrams for imaging advice, and past and present Strome lab members for feedback and helpful discussions. Some strains were provided by the CGC, which is funded by NIH P40 OD010440. This work used the Vincent J. Coates Genomics Sequencing Laboratory at UC Berkeley, supported by NIH S10 OD018174. The research conducted in this study was supported by NIH T32 GM008646, NIH F31 GM120882, and a STARS re-entry scholarship sponsored by Marilyn C. Davis \& the American Association of University Women to K.R.K. and by NIH R01 GM34059 to S.S. The content is solely the responsibility of the authors and does not necessarily represent the official views of the National Institutes of Health.

\section{Author contributions}

K.R.K. and S.S. designed the experiments, K.R.K. performed the experiments, A.R. processed RNA sequencing data, K.R.K. and A.R. performed bioinformatic analysis, K.R.K. and S.S. wrote the manuscript.

\section{Additional information}

Supplementary Information accompanies this paper at https://doi.org/10.1038/s41467 019-09141-w.

Competing interests: The authors declare no competing interests.

Reprints and permission information is available online at http://npg.nature.com/ reprintsandpermissions/

Journal peer review information: Nature Communications thanks Oded Rechavi and the other anonymous reviewers for their contribution to the peer review of this work. Peer reviewer reports are available.

Publisher's note: Springer Nature remains neutral with regard to jurisdictional claims in published maps and institutional affiliations. (c) Open Access This article is licensed under a Creative Commons
Attribution 4.0 International License, which permits use, sharing, adaptation, distribution and reproduction in any medium or format, as long as you give appropriate credit to the original author(s) and the source, provide a link to the Creative Commons license, and indicate if changes were made. The images or other third party material in this article are included in the article's Creative Commons license, unless indicated otherwise in a credit line to the material. If material is not included in the article's Creative Commons license and your intended use is not permitted by statutory regulation or exceeds the permitted use, you will need to obtain permission directly from the copyright holder. To view a copy of this license, visit http://creativecommons.org/ licenses/by/4.0/.

(c) The Author(s) 2019 